\title{
Evaluating the Semantic Web: A Task-Based Approach
}

\author{
Marta Sabou ${ }^{1}$, Jorge Gracia ${ }^{2}$, Sofia Angeletou ${ }^{1}$, \\ Mathieu d'Aquin ${ }^{1}$, and Enrico Motta ${ }^{1}$ \\ ${ }^{1}$ Knowledge Media Institute (KMi), The Open University, United Kingdom \\ \{r.m.sabou, s.angeletou, m.daquin, e.motta\}@open.ac.uk \\ ${ }^{2}$ IIS Department,University of Zaragoza, Spain \\ jogracia@unizar.es
}

\begin{abstract}
The increased availability of online knowledge has led to the design of several algorithms that solve a variety of tasks by harvesting the Semantic Web, i.e., by dynamically selecting and exploring a multitude of online ontologies. Our hypothesis is that the performance of such novel algorithms implicitly provides an insight into the quality of the used ontologies and thus opens the way to a task-based evaluation of the Semantic Web. We have investigated this hypothesis by studying the lessons learnt about online ontologies when used to solve three tasks: ontology matching, folksonomy enrichment, and word sense disambiguation. Our analysis leads to a suit of conclusions about the status of the Semantic Web, which highlight a number of strengths and weaknesses of the semantic information available online and complement the findings of other analysis of the Semantic Web landscape.
\end{abstract}

\section{Introduction}

The recent growth of the Semantic Web [19] and the appearance of semantic search engines such as Swoogle [1] and WATSON 9] that allow quick access to online knowledge has had a considerable impact on the design of Semantic Web applications. Indeed, there is a trend to move away from applications relying on a small amount of manually selected semantic sources towards a new generation of Semantic Web tools which dynamically select, reuse and combine a multitude of heterogeneous, online available ontologies 2021]. This paradigm of harvesting the Semantic Web has also inspired novel ways of performing a variety of tasks. For example, Alani [1] proposes a method for ontology learning that relies on cutting and pasting modules from online ontologies relevant to a set of keywords. In 14 the authors rely on online ontologies to disambiguate the senses of keywords used in a search engine query. Dynamically selected online ontologies play the role of background knowledge in ontology matching [26]27] or can be used to semantically enrich folksonomy tag spaces [431]. The experimental evaluations of these algorithms 41427. are still at an early stage, but, nevertheless, they provide strong evidence that the Semantic Web has reached a 
critical point where it can be used as a valuable source of knowledge to perform a variety of tasks.

Our hypothesis is that an important benefit of such novel algorithms lies in their potential use for evaluating the Semantic Web. Indeed, because they reuse a multitude of online ontologies, they can provide valuable insights into the qualitative aspects of these ontologies such as their suitability for a task, the properties of their vocabularies or the quality of their conceptual structure.

Such a task-based evaluation of the Semantic Web complements current efforts for evaluating (online) ontologies. Ontology evaluation has been a core research topic from the early stages of the Semantic Web leading to a set of approaches 6 6] distributed in two major categories. On the one side, a few approaches exist, which are based on the manual assessment of a set of ontology design criteria (e.g., OntoClean [15]). On the other side, there are many automatic approaches, which evaluate different aspects of an ontology (e.g., vocabulary, conceptual structure) by relying on different views of what constitutes a good "quality" ontology [28]. For example, the quality of an automatically learnt ontology can be judged in terms of its similarity to a manually constructed ontology or to the corpus from which it was extracted. Or, adopting a task-based view, the quality of an ontology can also be judged with respect to the performance of a task that uses it [25.

With the growth of the Semantic Web, the focus of ontology evaluation efforts has shifted towards online ontologies. Ontology selection methods [28] rely on evaluating ontology aspects such as popularity [71123, similarity to a domain or set of keywords [2/711/23] and the richness of the internal structure [2/7]. Furthermore, several overviews of the Semantic Web as a whole focus on the totality of online ontologies. Existing studies assess the size and growth rate of online knowledge [1019], as well as emerging trends in the adoption and use of representation languages and their primitives [51834]. While these findings are important, they do not give an insight into the suitability of online ontologies to be used for certain tasks. Hence, inspired by the paradigm introduced in 25], we propose to perform a task-based evaluation of the Semantic Web by analyzing the performance of novel algorithms that harvest it.

We test the feasibility and usefulness of such a task based evaluation approach by detailing the lessons we have learnt about the quality of online ontologies when they were employed to solve three different tasks: ontology matching (Section 2), folksonomy tagspace enrichment (Section 3) and query disambiguation (Section 4). We conclude in Section 5 with a number of observations about the status of the Semantic Web that support our hypothesis and are complementary to findings provided by similar studies of online ontologies [5/810 19 34].

\section{Case Study 1 - Ontology Matching}

Ontology matching is the task of determining the relations that hold between the entities of two ontologies 30 . In 26 we proposed a new paradigm to ontology matching which relies on harvesting the Semantic Web: it derives semantic mappings by dynamically selecting, exploiting, and combining multiple and 
heterogeneous online ontologies. For example, when matching two concepts labeled Researcher and AcademicStaff, the matcher would 1) identify (at run-time, during matching) online ontologies that can provide information about how these two concepts inter-relate and then 2) combine this information to infer the mapping. We distinguish two different strategies for deriving mappings [26]. In strategy S1 the mapping can be provided by a single ontology (e.g., stating that Researcher $\sqsubseteq$ AcademicStaff ). In strategy S2 a mapping can be derived by reasoning with information spread over several ontologies (e.g., that Researcher $\sqsubseteq$ ResearchStaff in one ontology and that ResearchStaff $\sqsubseteq$ AcademicStaff in another). We performed a large scale investigation and evaluation of this matching paradigm in 27] which provided a variety of insights into the quality of online ontologies as described next.

\subsection{Experimental Data and Results}

For experimental purposes we used two large, real life thesauri1. The United Nations Food and Agriculture Organization (FAO)'s AGROVOC thesaurus, version May 2006, consists of 28.174 descriptor terms (i.e., preferred terms) and 10.028 non-descriptor terms (i.e., alternative terms). The United States National Agricultural Library (NAL) Agricultural thesaurus NALT, version 2006, consists of 41.577 descriptor terms and 24.525 non-descriptor terms. We used both alternative and preferred terms in our experiments.

The matching process performed by using strategy S1 (see implementation details in [26]) resulted in a total of 6687 mappings (2330 subclass, 3710 superclass and 647 disjoint relations) obtained by dynamically selecting, exploring and combining 226 online ontologies. Fig. 1 shows the contribution of each of these ontologies to the alignment in terms of the number of mappings to which each ontology contributed and the percentage that this number represents.

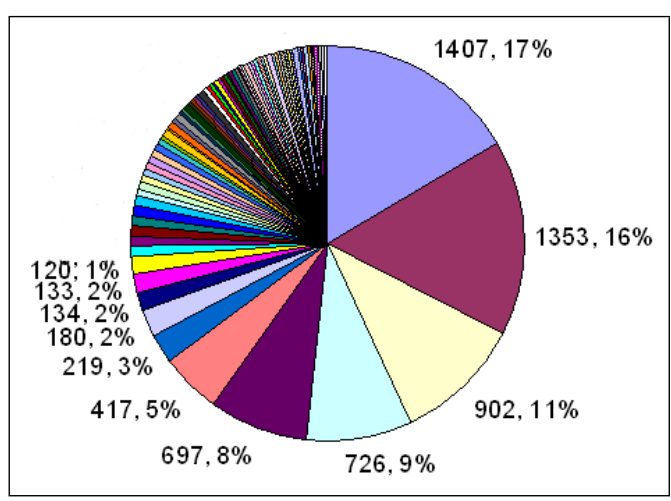

Fig. 1. Contribution of the online ontologies used by $\mathrm{S} 1$ to the alignment

Conclusion C1: Online ontologies are useful for the matching task. Based on these results, we can already conclude that online ontologies are useful to solve real life matching tasks. Indeed, if combined appropriately, they can provide a large amount of mappings between the matched ontologies. Moreover, in the next section we will show that the quality of the knowledge provided by the Semantic Web allows us to produce a performance comparable with the best performers among alternative approaches to ontology matching.

\footnotetext{
${ }^{1}$ This data set was used in the "OAEI'06 food Thesaurus Mapping Task",
} http://www.few.vu.nl/ wrvhage/oaei2006/ 
Table 1. Evaluation results

\begin{tabular}{|l|c|c|c|}
\hline & Group 1 & Group 2 & Agreed by All \\
\hline Correct & 586 & 666 & 525 \\
\hline False & 346 & 299 & 217 \\
\hline Don't know & 68 & 35 & 10 \\
\hline Precision & $\mathbf{6 3 \%}$ & $\mathbf{6 9 \%}$ & $\mathbf{7 0 \%}$ \\
\hline
\end{tabular}

\subsection{Quality of Online Ontologies}

According to 25, the essence of a task based evaluation is that the quality of an ontology correlates with the performance of the task in which it is employed. In the case of matching this means that the precision of the alignment is an indication of the quality of online ontologies explored to derive it.

To assess the quality of the knowledge provided by online ontologies we performed a manual assessment of 1000 mappings (i.e., 15\% of the alignment). We relied on six members of our lab working in the area of the Semantic Web, and thus familiar with ontologies and ontology modeling. We performed two parallel evaluations of the sample mappings (i.e., each mapping has been evaluated by two different evaluators). The participants evaluated each mapping as Correct, False or "Don't know" when they could not judge the correctness of the statement. We computed the precision of the obtained alignment as the ratio of Correct mappings over all the evaluated mappings (i.e., those evaluated either as Correct or False) and obtained precision values of $63 \%$ and $69 \%$ for the two groups (see Table 1). To level out any differences, we also computed the precision of the fraction of the alignment on which both groups agreed (i.e., 742 mappings, $74 \%$ ). We consider the so obtained precision value of $70 \%$ as a typical baseline performance that can be achieved by harvesting online knowledge.

A manual inspection of the 217 false mappings on which both groups agreed revealed that 114 (i.e., 53\%) are due to our simplistic anchoring mechanism (i.e., finding concepts in online ontologies that correspond to the matched concepts). For example, in Table 2 c_6443 labeled with Rams and referring to an uncastrated adult male sheer 2 is put in correspondence with a similarly labeled concept (ram), but which means Random Access Memory in the context of the online ontology. An anchoring mechanism that could prevent us from deriving these false mappings (thus reducing their number to 103) will imply an increase in precision from $70 \%$ to $87 \%$.

To fully understand the significance of these values, it is important to compare them to the performance of other background knowledge based techniques. However, the precision values we found in the literature were reported on different data sets, therefore we can consider them only as indicative, and in addition only S-Match reports on recall values [13. The technique of Aleksovski et al. was evaluated on a Gold Standard of mappings for 200 concepts and produced a precision of $76 \%$ [3]. The matching techniques proposed by van Hage et al. reach precision values of $53 \%-75 \%$ when exploring a domain specific textual

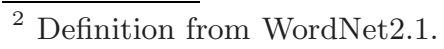


Subsumption used to model generic relations. One of the most common errors was the use of subsumption as a way to model the fact that there exists some type of relation between two concepts, e.g., Survey $\sqsubseteq$ Marketing, Irrigation $\sqsubseteq$ Agriculture, Biographies $\sqsubseteq$ People. This case leads to 40 false mappings (i.e., $18 \%$ ).

Subsumption used to model part-whole relations. Subsumption is also used in several ontologies to model part-whole relations. These ontologies resulted in 16 (7\%) incorrect mappings, e.g., Branch $\sqsubseteq$ Tree, Leaf $\sqsubseteq$ Plant.

Subsumption used to model roles. We found 11 false mappings (5\%) derived because roles were incorrectly modeled as subclass relations, for example, that Garlic, Leek Ingredient (in fact, Leek is a Vegetable but in some contexts it plays the the role of an ingredient).

Inaccurate labeling. We also found 12 cases (5\%) when a correct subclass relation introduced errors due to the inaccurate labeling of its concepts. For example, $\mathrm{O}_{1}{ }^{3}$ states that coal $\sqsubseteq$ industry, where coal refers to coal industry rather than the concept of Coal itself. Similarly, for Database Enzime in $O_{1} 4$, Enzyme refers to an enzyme database rather than describing the class of all enzymes.

Different Views. Finally, some of the explored ontologies adopted a certain view on the relation of two concepts that was not in concordance with the context of the mapping and/or the perspective of the evaluators. For example, TAP considers lobsters kinds of Fishes, a perspective with which none of the evaluators agreed.

Conclusion C3: Online ontologies contain modeling errors which hamper the quality of the alignment. Most errors are due to the incorrect use of subsumption to model generic relations, roles and meronymy.

\subsection{Contradictory Statements in Online Ontologies}

The novelty of techniques that harvest the Semantic Web lies in their ability to combine information from multiple, different ontologies. As such, they need to deal with potentially contradictory information supplied by different sources. For example, in the case of ontology matching, contradictory mapping relations could be derived between two concepts by relying on different ontologies. The question is how frequent this phenomenon is, i.e., do different online ontologies lead to contradictory mappings between two given terms?

To answer this question, we ran a modified variant of S1: for every pair of concept labels we derive mappings from all the online ontologies that mention them. While we have discovered mappings between a high number of label pairs (6425), the number of cases when contradictory mappings are derived is surprisingly low and accounts to only six pairs (see Table 3 ).

\footnotetext{
3 http://www . aifb.uni-karlsruhe.de/WBS/meh/mapping/data/russia1a.rdf

4 http://mensa.sl.iupui.edu/ontology/Database.owl
} 
Table 3. Contradictory statements in online ontologies

\begin{tabular}{|c|c|c|c|c|}
\hline $\begin{array}{c}\text { AGROVOC } \\
\text { label }\end{array}$ & NALT & $\begin{array}{c}\text { Nr. Subclass } \\
\text { label }\end{array}$ & $\begin{array}{c}\text { Nr. Superclass } \\
\text { relations }(\stackrel{ }{\longrightarrow})\end{array}$ & $\begin{array}{c}\text { Nr. Disjunct } \\
\text { relations }(\stackrel{\sqsupseteq}{\longrightarrow})\end{array}$ \\
\hline fruit & tomato & 0 & 3 & 1 \\
\hline sea & river & 0 & 1 & 2 \\
\hline energy & light & 0 & 1 & 1 \\
\hline meat & seafood & 0 & 2 & 12 \\
\hline mushroom & pizza & 1 & 0 & 1 \\
\hline sea & ocean & 1 & 1 & 0 \\
\hline
\end{tabular}

Conclusion C4: Only few online ontologies contain contradictory relations between two given concepts. Our preliminary observations indicate that the correct mapping can normally be filtered out with simple statistical means: the most frequently derived relation is likely to be correct.

\subsection{Inconsistencies in Multiple Mappings Drawn from Different Ontologies}

In the previous section we have only looked at a rather basic form of contradiction, where contradictory relations have been explicitly stated between two items. As pointed out, these cases appear to be very infrequent. However, if we go beyond relations between two items and look at a number of mappings as a whole, then inconsistencies arise more frequently. Fig. 2 provides such an example, where Vegetable $_{i}$ is discovered to be disjoint with Fruit_(Food $)_{j}$, Tomatoes $_{j}$ is a

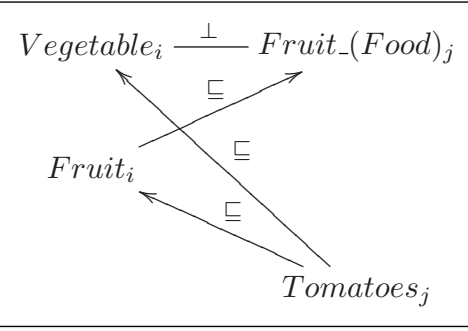

Fig. 2. Example of incoherence in mappings subclass of both concepts and thus unsatisfiable: there cannot be any instance of Tomatoes ${ }_{j}$, since it would have to belong to two disjoint classes at the same time.

As already said, this phenomenon of generating sets of inconsistent mappings was more pronounced than expected. Indeed, our automatic incoherence detection mechanism has identified 306 base incoherences that corrupted the entire alignment. A few mappings between very generic concepts with many subclasses (e.g., Foods $\sqsubseteq$ Products, People $\sqsubseteq$ Agents) caused the majority of the incoherences. Fortunately, these can be isolated and disposed of automatically using reasoning mechanisms, thus leading to the improvement of the entire alignment.

Conclusion C5: Because different ontologies rely on different views or different contexts, they may contain contradictory information, leading to inconsistent sets of mappings. 


\section{Case Study 2 - Folksonomy Tagspace Enrichment}

Social tagging system 5 are highly successful due to the ease of the tagging process: users need neither to have prior knowledge or specific skills to use them 18 35, nor to rely on a priori agreed structure or shared vocabulary. While folksonomies (i.e., lightweight structures that emerge from the tag space) are easy to create, they only weakly support content retrieval since they are agnostic to the relations between their tags: a search for mammal ignores all resources that are not tagged with this specific word, even if they are tagged with semantically related terms such as lion, cow, cat. Most approaches which address this problem 122935] identify clusters of implicitly related tags (e.g., that mammal and lion are related). Specia and Motta 31 go one step further by proposing to make the semantic relations between tags explicit (e.g., that mammal is more generic than lion). They envision a semantic enrichment algorithm which complies with the paradigm of harvesting the Semantic Web by dynamically exploring and combining multiple online ontologies to derive explicit relations among implicitly interrelated tags.

A simplified version of the enrichment algorithm has been experimentally investigated in 4 by relying on the same implementation of relation discovery as used for ontology matching in 26] (i.e., strategy S1). Given a set of implicitly related tags, the prototype identifies subsumption and disjointness relations between them and constructs

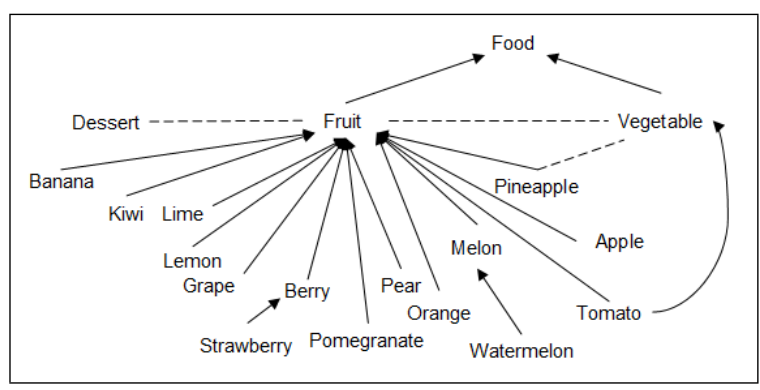

Fig. 3. Semantically enriched tag cluster for Fruit a semantic structure based on these relations. The first experiments on tag sets identified in 31 led to suboptimal results due to (1) the small size of the clusters (3-5 tags), (2) the low coverage of certain tag types in online ontologies and (3) the limitation of the software (it only identifies subsumption relations while most tags were related through generic relations). Therefore, we ran a second set of experiments on larger tag clusters identified with the Flickr AP 6 around a handful of terms from domains that are well-covered by online ontologies. In these cases the process resulted in rich knowledge structures, such as for the tag cluster of Fruit in Fig. 3 (dotted lines denote disjointness). The general conclusion of the study is that while online ontologies can indeed be used to semantically enrich folksonomies, some of their characteristics hamper the process, as described next.

${ }^{5}$ E.g., Flickr (http://flickr.com/), del.icio.us (http://del.icio.us/

6 http://www.flickr.com/services/api/flickr.tags.getRelated.html 


\section{Conclusion C6: Online ontologies only weakly cover certain tag cate- gories, as follows:}

Novel terminology. Folksonomies are social artifacts built by large masses of people. They dynamically change to reflect the latest terminology in several domains and therefore greatly differ from ontologies which are usually developed by small groups of people and evolve much slower. As such, it is not surprising that many of the tags used in folksonomies, e.g., \{ajax, css $\}$, have not yet been integrated into ontologies. Identifying such novel terminology has a great potential for the Semantic Web as it could represent a first step towards updating existing ontologies.

Scientific terminology (e.g., plant taxonomy) can only rarely be found in online ontologies. This could change however if large ontologies containing such information (e.g., AGROVOC) would be made available online.

Multilingual tags. Both Flickr and Del.icio.us (but especially Flickr) contain tags from a variety of languages and not only English. These tags are usually hard to find on the Semantic Web because the language coverage of the existing ontologies is rather low. Indeed, statistic 8 performed on a large collection of online ontologies (1177) in the context of the OntoSelect library [7] indicate that $63 \%$ of these ontolgies contain English labels, while a much smaller percentage contains labels in other languages (German $13.25 \%$, French $6.02 \%$, Portuguese 3.61\%, Spanish 3.01\%).

Photographic jargon. Because Flickr is a photo annotation and sharing site, many tags reflect terms used in photography, such as \{nikon, canon, closeup\}. Unfortunately, this domain is weakly covered in the Semantic Web.

Our study also found that, like in the case of ontology matching (C5), online ontologies can reflect different views and when used in combination can lead to inconsistencies in the derived structures. For example, the structure in Fig. 3 states that Fruit is disjoint with Dessert. The validity of this statement depends on the point of view we adopt since some would argue that fruits are desserts. Such different views can have more serious consequences. For example, Tomato is considered to be both a Fruit and a Vegetable. The first statement is valid in a biological context, since a tomato is the fruit of a tomato plant, however, normally one would classify tomatoes as types of vegetables. While such different views can co-exist, the fact that Fruit and Vegetable are disjoint makes the derived semantic structure inconsistent.

\section{Case Study 3 - Word Sense Disambiguation}

The goal of the Word Sense Disambiguation (WSD) task is to identify the appropriate sense of a word in a given context. Usually this task involves identifying a set of possible senses and then filtering out the right one based on some similarity algorithms. Existing approaches [17/22/24] exploit a given lexical resource

\footnotetext{
${ }^{7}$ At the time of our experiments, March 2007.

8 http://olp.dfki.de/OntoSelect/w/index.php?mode=stats
} 
(mainly WordNet) or ontology (small set of ontologies) as sources of word senses and then rely on one or more particular ontologies or corpora to compute semantic measures. Because they require the selection of appropriate knowledge sources a priori, these approaches are not suitable in cases when the domain of the words to be disambiguated is determined at run time. This limitation is addressed by a novel, unsupervised, multi-ontology WSD method [14 which 1) relies on dynamically identified online ontologies as sources for candidate word senses and 2) employs algorithms that combine information available both on the Semantic Web and the Web in order to compute semantic measures.

For example, suppose that we want to disambiguate Java in the context of "Indonesia Java". In a first step, the algorithm identifies a set of possible senses 9 for each keyword by exploring online ontologies 10 and combines highly similar senses to avoid redundancies. Table 4 shows the candidate senses for Java and their characterization by their synonyms (i.e., Level 0) and superterms (Level 1, in this case direct hypernyms). A second, disambiguation step consists in computing a Google based semantic relatedness between Indonesia and each involved term (e.g., Indonesia $\leftrightarrow$ Java, Indonesia $\leftrightarrow$ Island) and combining the obtained values into a final $[0,1]$ range score. The highest score indicates the most appropriate sense, i.e., Java $\sqsubseteq$ Island in our case. While the large scale evaluation of this method is still in progress, we can already summarize some qualitative conclusions about the characteristics of online ontologies.

Table 4. Disambiguation of Java in the context of "Indonesia Java"

\begin{tabular}{|c|c|c|c|c|}
\hline \#Sense & Type & Level 0 & Level 1 & Score \\
\hline $\mathbf{1}$ & concept & Java & island & $\mathbf{0 . 3 8 7}$ \\
\hline $\mathbf{2}$ & concept & java, coffee & drink & 0.251 \\
\hline $\mathbf{3}$ & concept & java & programming language & 0.116 \\
\hline
\end{tabular}

\section{Conclusion C7: Online ontologies provide a good source for word sense}

definitions. A major benefit of relying on multiple, online ontologies is that a much larger set of keyword senses can be discovered than in cases when few, predefined resources are used. For example, many traditional methods fail to disambiguate developer in "UML handbook for developers" because WordNet2.1 does not contain the word UML (acronym of Unified Modeling Language), neither the intended meaning of developer as someone who develops software. This information is however available in online ontologies: $U M L$ is a concept in the Book11 ontology (subsumed by SoftwareDesigns), and developer is a property described as "Developer of software" in the DOAP 12 ontology. As evident from

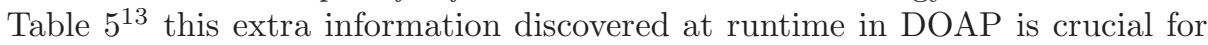
identifying the appropriate sense for developer.

\footnotetext{
${ }^{9}$ Defined by the ontological context of the term: synonyms, hypernyms etc.

${ }^{10}$ In addition to WordNet or any other local resource.

11 http://islab.hanyang.ac.kr/damls/Book.daml

12 http://usefulinc.com/ns/doap

13 Level 1 here contains direct hypernyms for concepts and domains for properties.
} 
Table 5. Disambiguation of developer in the context "UML handbook developer"

\begin{tabular}{|c|c|c|c|c|c|c|}
\hline \#Sense & Type & Source & Description & Level 0 & Level 1 & Score \\
\hline 1 & property & DOAP & $\begin{array}{c}\text { "Developer of software } \\
\text { for the project" }\end{array}$ & developer & project & $\mathbf{0 . 2 9 3}$ \\
\hline 2 & concept & WordNet & $\begin{array}{c}\text { "photographic } \\
\text { equipment ..." }\end{array}$ & developer & $\begin{array}{c}\text { photographic } \\
\text { equipment }\end{array}$ & 0.239 \\
\hline 3 & concept & WordNet & $\begin{array}{c}\text { "someone who develops } \\
\text { real estate" }\end{array}$ & developer & creator & 0.230 \\
\hline
\end{tabular}

Conclusion C8: Disambiguation results are influenced by modeling errors in online ontologies. However, due to its nature, the algorithm, is only partially affected by the typical ontology errors described in Section 2.3 (see Table 6). Indeed, the disambiguation algorithm uses part of the ontological context that characterizes a sense (e.g., subsumption, generic relations) in order to restrict the semantic field of the sense and to distinguish it from other senses of the same word. Such ontological information is used as a basis for relatedness computation and not exploited through formal reasoning as in the case of ontology matching. Therefore, the algorithm is not affected by the quality of formal modeling. For example, to characterize branch in its biological sense, an incorrectly modeled part-whole relation (Branch $\sqsubseteq$ Tree) could lead to the same disambiguation result as using a correct subsumption (Branch $\sqsubseteq$ Stalk). Also, agriculture could be an acceptable context to distinguish irrigation as supplying dry land with water from its medical sense. We conclude that error types 1 and 2 do not affect intrinsically the algorithm. On the other hand, the last three types of errors which associate a given term with other terms that do not reflect its sense have a major influence on the algorithm. For example, in Enzyme $\sqsubseteq$ Database, the inaccurate labeling could give unpredictable results in the computed semantic measures. Also, the user could have different views from some online ontologies (error 5) and thus obtain an undesired result.

Table 6. Sensitivity of disambiguation algorithm to frequent ontology errors

\begin{tabular}{|l|c|c|c|}
\hline & Error Type & Example & Effect on algorithm? \\
\hline $\mathbf{1}$ & Subsumption as generic relation & Irrigation $\sqsubseteq$ Agriculture & No \\
\hline $\mathbf{2}$ & Subsumption as part-whole & Branch $\sqsubseteq$ Tree & No \\
\hline $\mathbf{3}$ & Subsumption as role & garlic $\sqsubseteq$ ingredient & Yes \\
\hline $\mathbf{4}$ & Inaccurate labeling & enzyme $\sqsubseteq$ database & Yes \\
\hline $\mathbf{5}$ & Different view & lobster $\sqsubseteq$ fish & Yes \\
\hline
\end{tabular}

\section{Conclusion C9: Many online ontologies have a weak internal structure} and thus hamper the performance of the method. For example, few online ontologies contain synonyms or non-taxonomic relations. We even found ontologies containing no relations at all. As a result, our algorithm can identify richly (e.g., extracted from WordNet) as well as poorly defined senses for the same word. Such uneven semantic characterization has a negative effect on the algorithm 
(which was built to compare similarly rich descriptions of senses) and can lead to suboptimal results. This insight in the general quality of online ontologies lead us to envision two important future changes. First, our tool should only rely on senses extracted from semantically rich ontologies which could be identified using a ranking mechanism such as AKTiveRank 2. Second, the semantic measures we use should adapt to ontological contexts of variable richness (e.g., glosses should be given a high importance in ontologies with a poor taxonomy but rich in descriptions).

Conclusion C10: Parsing errors and broken links further hamper the functioning of the method. For example, from the 602 online ontologies identified for describing 25 terms randomly extracted from a list of frequently used keyword 14 , $252(42 \%)$ could not be correctly parsed into Jena 15 models due to parsing errors or broken links. Without being conclusive, this limited example illustrates the proportion of the problem.

\section{Conclusions and Future Work}

The hypothesis put forward in this paper is that novel algorithms which harvest online knowledge can facilitate a task based evaluation of the Semantic Web. Accordingly, we report on quality characteristics of online ontologies determined by analyzing the experimental results of three algorithms which solve divers tasks: ontology matching, folksonomy enrichment and WSD.

The major conclusion that we derive based on the content of our observations is that online ontologies have a great potential for being used in combination to solve a variety of real life tasks. Indeed, combining knowledge from multiple ontologies lead to a broad range of high quality mappings (C1) and to more word sense definitions during WSD (C7). In the case of ontology matching, we could also experimentally prove that the obtained alignment had a high precision, despite relying on more than 200 ontologies (C2). There are, however, some undesired effects caused by combining knowledge from multiple sources. Even if only in very few cases, contradicting statements can be obtained about two given concepts (C4). Then, the first two case studies were affected by the fact that online ontologies often reflect different views which can lead to incoherent knowledge structures when combined (C5). Overall, however, these findings deliver an important message: even at this early stage of development, the Semantic Web is a powerful source of background knowledge that can be exploited to successfully tackle real world tasks.

Besides providing task-centric conclusions, our approach also lead to observations about other aspects of online ontologies. At a syntactic level, several ontologies cannot be accessed due to parsing errors and broken links (C10). Second, regarding their vocabularies, online ontologies provide a weak coverage of certain types of folksonomy tags, such as novel terms, multilingual tags or scientific terms (C6). Third, we gained insight into major issues with the quality

\footnotetext{
14 http://www.google.com/press/zeitgeist_monthly.html

15 http://jena.sourceforge.net/
} 
of the knowledge structures of online ontologies. We found that many have a weak (or no) structure and thus hampered the WSD method (C9). Even more worryingly, we identified a set of modeling errors, mostly related to the misuse of subsumption relations, which affected (to different degrees) both the formal reasoning based matching algorithm (C3) and the WSD process (C8).

While our conclusions provide a better understanding of the current state of the Semantic Web (complementary with the conclusions of other similar studies [58101934]), they could further benefit the research community as follows. First, we consider them as a proof that a task based evaluation is feasible and useful, thus supporting the hypothesis of the paper. Therefore, we wish to provide a more formal model for performing evaluations in this manner. Second, our findings have highlighted the need for novel evaluation methods that are capable to automatically identify more subtle characteristics such as the quality of the modeling [33. Finally, these findings are valuable knowledge for those who wish to (re-)design algorithms that harvest the Semantic Web in a way that they maximally benefit from this rich and growing online knowledge repository.

Acknowledgements. This work was funded by the OpenKnowledge IST-FF6027253 and NeOn IST-FF6-027595 projects, the CICYT TIN2004-07999-C02-02 project and by the Government of Aragon-CAI grant IT11/06.

\section{References}

1. Alani, H.: Position Paper: Ontology Construction from Online Ontologies. In: Proc. of WWW (2006)

2. Alani, H., Brewster, C., Shadbolt, N.: Ranking Ontologies with AKTiveRank. In: Cruz, I., Decker, S., Allemang, D., Preist, C., Schwabe, D., Mika, P., Uschold, M., Aroyo, L. (eds.) ISWC 2006. LNCS, vol. 4273, Springer, Heidelberg (2006)

3. Aleksovski, Z., Klein, M., ten Katen, W., van Harmelen, F.: Matching Unstructured Vocabularies using a Background Ontology. In: Staab, S., Svátek, V. (eds.) EKAW 2006. LNCS (LNAI), vol. 4248, Springer, Heidelberg (2006)

4. Angeletou, S., Sabou, M., Specia, L., Motta, E.: Bridging the Gap Between Folksonomies and the Semantic Web: An Experience Report. In: Proc. of the ESWC Bridging the Gap between Semantic Web and Web 2.0 Wokshop (2007)

5. Bechhofer, S., Volz, R.: Patching Syntax in OWL ontologies. In: McIlraith, S.A., Plexousakis, D., van Harmelen, F. (eds.) ISWC 2004. LNCS, vol. 3298, Springer, Heidelberg (2004)

6. Brank, J., Grobelnik, M., Mladenic, D.: A survey of ontology evaluation techniques. In: Proc. of the Conf. on Data Mining and Data Warehouses (2005)

7. Buitelaar, P., Eigner, T., Declerck, T.: OntoSelect: A Dynamic Ontology Library with Support for Ontology Selection. In: McIlraith, S.A., Plexousakis, D., van Harmelen, F. (eds.) ISWC 2004. LNCS, vol. 3298, Springer, Heidelberg (2004)

8. d'Aquin, M., Baldassarre, C., Gridinoc, L., Angeletou, S., Sabou, M., Motta, E.: Characterizig Knowledge on the Semantic Web with WATSON, (submitted for peer review) (2007)

9. d'Aquin, M., Sabou, M., Dzbor, M., Baldassarre, C., Gridinoc, L., Angeletou, S., Motta, E.: WATSON: A Gateway for the Semantic Web. In: ESWC Poster (2007) 
10. Ding, L., Finin, T.: Characterizing the Semantic Web on the Web. In: Cruz, I., Decker, S., Allemang, D., Preist, C., Schwabe, D., Mika, P., Uschold, M., Aroyo, L. (eds.) ISWC 2006. LNCS, vol. 4273, Springer, Heidelberg (2006)

11. Ding, L., Pan, R., Finin, T., Joshi, A., Peng, Y., Kolari, P.: Finding and Ranking Knowledge on the Semantic Web. In: Gil, Y., Motta, E., Benjamins, V.R., Musen, M.A. (eds.) ISWC 2005. LNCS, vol. 3729, Springer, Heidelberg (2005)

12. Begelman, G., Keller, P., Smadja, F.: Automated Tag Clustering: Improving search and exploration in the tag space. In: WWW 2006. Proc. of the Collaborative Web Tagging Workshop (2006)

13. Giunchiglia, F., Shvaiko, P., Yatskevich, M.: Discovering Missing Background Knowledge in Ontology Matching. In: ECAI 2006. LNCS (LNAI), vol. 4386, Springer, Heidelberg (2006)

14. Gracia, J., Trillo, R., Espinoza, M., Mena, E.: Querying the Web: A Multiontology Disambiguation Method. In: Proc. of ICWE (2006)

15. Guarino, N., Welty, C.A.: An Overview of OntoClean. In: Staab, S., Studer, R. (eds.) Handbook on Ontologies, Springer, Heidelberg (2004)

16. Hartmann, J., Sure, Y., Giboin, A., Maynard, D., Suarez-Figueroa, M.C., Cuel, R.: Methods for ontology evaluation. Knowledge Web Deliverable. In: Methods for ontology evaluation. Knowledge Web Deliverable D1.2.3 (2005)

17. Hassell, J., Aleman-Meza, B., Arpinar, I.B.: Ontology-Driven Automatic Entity Disambiguation in Unstructured Text. In: Cruz, I., Decker, S., Allemang, D., Preist, C., Schwabe, D., Mika, P., Uschold, M., Aroyo, L. (eds.) ISWC 2006. LNCS, vol. 4273, Springer, Heidelberg (2006)

18. Hotho, A., Jaschke, R., Schmitz, C., Stumme, G.: Information Retrieval in Folksonomies: Search and Ranking. In: Sure, Y., Domingue, J. (eds.) ESWC 2006. LNCS, vol. 4011, Springer, Heidelberg (2006)

19. Lee, J., Goodwin, R.: The Semantic Webscape: a View of the Semantic Web. IBM Research Report (2004)

20. Motta, E., Sabou, M.: Language Technologies and the Evolution of the Semantic Web. In: Proc. of the Int. Conf. on Language Resources and Evaluation (2006)

21. Motta, E., Sabou, M.: Next Generation Semantic Web Applications. In: Mizoguchi, R., Shi, Z., Giunchiglia, F. (eds.) ASWC 2006. LNCS, vol. 4185, Springer, Heidelberg (2006)

22. Navigli, R., Velardi, P.: Structural Semantic Interconnections: A Knowledge-Based Approach to Word Sense Disambiguation. IEEE Trans. Pattern Anal. Mach. Intell. 27(7), 1075-1086 (2005)

23. Patel, C., Supekar, K., Lee, Y., Park, E.K.: OntoKhoj: A Semantic Web Portal for Ontology Searching, Ranking and Classification. In: Proc. of WIDM (2003)

24. Pedersen, T., Banerjee, S., Patwardhan, S.: Maximizing Semantic Relatedness to Perform Word Sense Disambiguation, 2005. Research Report UMSI 2005/25

25. Porzel, R., Malaka, R.: A Task-based Approach for Ontology Evaluation. In: Proc. of the ECAI Workshop on Ontology Learning and Population (2004)

26. Sabou, M., d'Aquin, M., Motta, E.: Using the Semantic Web as Background Knowledge for Ontology Mapping. In: Proc. of the Ontology Matching WS (2006)

27. Sabou, M.: M. d'Aquin, and E. Motta. Using the Semantic Web as Background Knowledge for Ontology Matching: Investigating a New Paradigm (submitted for peer review, 2007)

28. Sabou, M., Lopez, V., Motta, E., Uren, V.: Ontology Selection: Ontology Evaluation on the Real Semantic Web. In: Proc. of the EON Workshop (2006)

29. Schmitz, P.: Inducing Ontology from Flickr Tags. In: WWW 2006. Proc. of the Collaborative Web Tagging Workshop (2006) 
30. Shvaiko, P., Euzenat, J.: A Survey of Schema-based Matching Approaches. Journal on Data Semantics IV (2005)

31. Specia, L., Motta, E.: Integrating Folksonomies with the Semantic Web. In: ESWC 2007. LNCS, vol. 4519, Springer, Heidelberg (2007)

32. van Hage, W., Katrenko, S., Schreiber, G.: A Method to Combine Linguistic Ontology-Mapping Techniques. In: Gil, Y., Motta, E., Benjamins, V.R., Musen, M.A. (eds.) ISWC 2005. LNCS, vol. 3729, Springer, Heidelberg (2005)

33. Völker, J., Vrandecic, D., Sure, Y.: Automatic Evaluation of Ontologies (AEON). In: Gil, Y., Motta, E., Benjamins, V.R., Musen, M.A. (eds.) ISWC 2005. LNCS, vol. 3729, Springer, Heidelberg (2005)

34. Wang, T.D., Parsia, B., Hendler, J.A.: A Survey of the Web Ontology Landscape. In: Cruz, I., Decker, S., Allemang, D., Preist, C., Schwabe, D., Mika, P., Uschold, M., Aroyo, L. (eds.) ISWC 2006. LNCS, vol. 4273, Springer, Heidelberg (2006)

35. Wu, X., Zhang, L., Yu, Y.: Exploring Social Annotations for the Semantic Web. In: Proc. of WWW (2006) 